\title{
Advanced Non-Small-Cell Lung Cancer in Elderly Patients: Patient Features and Therapeutic Management
}

\author{
Nakano Takayuki (D), Tanimura Keiko, Uchino Junji (D), Kaneko Yoshiko ${ }^{D}$, \\ Tamiya Nobuyo $\sqrt{ }$, Yamada Tadaaki, and Takayama Koichi \\ Department of Pulmonary Medicine, Kyoto Prefectural University of Medicine, 465 Kajii-cho, Kawaramachi-Hirokoji, \\ Kamigyo-ku, Kyoto 602-8566, Japan \\ Correspondence should be addressed to Uchino Junji; uchino@koto.kpu-m.ac.jp
}

Received 27 October 2017; Revised 14 March 2018; Accepted 25 March 2018; Published 30 April 2018

Academic Editor: Nam Nguyen

Copyright ( $) 2018$ Nakano Takayuki et al. This is an open access article distributed under the Creative Commons Attribution License, which permits unrestricted use, distribution, and reproduction in any medium, provided the original work is properly cited.

\begin{abstract}
Lung cancer has the highest mortality rate among all cancers in most developed countries. The number of elderly patients with lung cancer has been increasing, reflecting the global increase in aging population. Therefore, standard chemotherapeutic regimens for elderly patients with lung cancer need to be established. However, the effectiveness of chemotherapy in elderly patients with advanced non-small-cell lung cancer remains controversial because they are often excluded from clinical trials. Some clinical trials have shown that the therapeutic benefit of a third-generation anticancer drug alone was superior to best supportive care. In contrast, platinum-doublet was superior only in terms of overall survival and progression-free survival, and other trials reported an increased rate of treatment-related death in the elderly patients. In recent years, some novel treatment modalities for lung cancer have been developed and shown to significantly improve the therapeutic outcomes, including targeted therapy for lung cancer harboring driver mutation, combination therapy of angiogenesis inhibitor and cytotoxic agents, and immune checkpoint inhibitor. Although several clinical trials with these agents have shown favorable outcome regardless of age, their safety in the elderly patients has not been established. Herein, we discuss the current clinical status and future prospects in elderly patients with lung cancer.
\end{abstract}

\section{Introduction}

Lung cancer is among the malignancies with poor prognosis. In 2015, lung cancer was the fifth leading cause of mortality, with the World Health Organization (WHO) reporting 1.7 million deaths worldwide [1]. This mortality rate was the highest among all cancers of the organs. Given that old age increases the risk for developing lung cancer, the proportion of elderly patients with lung cancer has also been increasing, reflecting the global increase in aging population [2]. Such trend is more prominent in Japan because of its high elderly population, and $75 \%$ of patients who died of lung cancer are the elderly aged 70 years or older [3]. Although elderly patients with lung cancer can also be treated with standard therapy, only few clinical trials target elderly patients. Thus, the therapeutic management for lung cancer has not been properly assessed for this patient group. Some clinical trials showed an increase in the incidence of adverse events and poor efficacy of standard treatment modalities; by contrast, other drugs were reported to achieve favorable antitumor effects in both elderly and young patients. However, most clinical trials that reported these promising studies excluded elderly patients with poor physical state [4].

In recent years, drug therapy for advanced lung cancer has rapidly progressed. Due to the relatively mild adverse events compared to conventional cytotoxic agents, drug therapy, such as molecular targeted drugs and immune checkpoint inhibitors, has been widely considered to be appropriate for elderly patients with lung cancer for whom therapy is indicated via biomarker testing [5]. In this study, we discuss the current state of and the issues to be addressed in drug therapy for elderly patients with advanced non-small-cell lung cancer (NSCLC). 


\section{Features of Elderly Patients with Lung Cancer}

Compared with young patients with cancer, the elderly has several characteristics that need to be considered during treatment, including reduced ability for performance of activities of daily living, history of multiple comorbid diseases, decline in organ function, reduction in cognitive function, and changes in social environment. In lung cancer, cigarette smoking (which is the leading cause of lung cancer) and chronic obstructive pulmonary disease (COPD, which is the most common comorbidity of lung cancer) often limit air flow, decreasing the ability for physical activity. The adverse effects of cigarette smoke accumulates, and the risk for COPD increases with age [6]. Moreover, elderly patients with lung cancer tend to have cardiac comorbidities such as congestive heart failure, which can be a problem in chemotherapy, particularly for chemotherapeutics that need to be administered with high-volume hydration [7]. Furthermore, Repetto et al. reported that the risk of cognitive disorders in patients with advanced lung cancer increases with age. The percentage of patients with low mini-mental state examination score is at $29.0 \%$ among those aged 74 years or younger versus $78.4 \%$ among those aged over 85 years [8].

These factors should be carefully considered in developing a chemotherapy plan for elderly patients with lung cancer.

\section{Changes in Physiological Function and Pharmacokinetics in the Elderly}

Organ and physiological functions decrease over time after the age of 40 years. Because drugs are excreted from the kidney or liver, pharmacokinetics are affected by decreases in renal or hepatic bioactivity.

Renal function is easily affected by decreased renal blood flow and arteriosclerotic change due to aging, and the rate of creatinine clearance becomes two times slower after the age of 45 years [9]. Therefore, proper dosage adjustment is required during drug administration in elderly patients.

Multiple factors, such as hepatic blood flow, metabolizing enzyme activity, and ratio of an unbound drug with plasma protein, affect the pharmacokinetics of drugs excreted mainly through the hepatic/biliary metabolism. Physiological changes in hepatic function, reductions in hepatic blood flow caused by reduced cardiac output, and decreases in the metabolizing enzyme CYP due to aging have been reported [10]. In addition, renal dysfunction significantly affects the functions of drug-metabolizing enzymes and transporters in the liver [11]. Given that elderly patients commonly develop multiple types of organ dysfunction, a reduced capacity for drug clearance can cause persistently high drug levels in the blood, which may lead to an increase in drug toxicity. Therefore, appropriate dosage adjustment and careful monitoring are required in this patient population.

\section{Chemotherapy for Elderly Patients with Advanced Non-Small-Cell Lung Cancer}

4.1. Cytotoxic Chemotherapy. In the 20th century, evidence on the efficacy of chemotherapy for advanced NSCLC in elderly patients was limited. However, the results of two phase III trials, namely, the Elderly Lung Cancer Vinorelbine Italian Study and the Multicenter Italian Lung Cancer in the Elderly Study (MILES), showed that the therapeutic benefits of a third-generation anticancer drug alone, such as vinorelbine (VNR) and gemcitabine (GEM), are superior to best supportive care alone $[12,13]$. In the phase III WJTOG9904 trial conducted in Japan, although no significant difference in outcomes was obtained, docetaxel (DTX) alone extended overall survival (OS) and progression-free survival (PFS) compared to VNR alone. Therefore, DTX has been recommended in Japan's guidelines for treatment of lung cancer in the elderly [14]. However, the most superior third-generation anticancer drug remains undetermined. After 2010, subgroup analysis of pemetrexed and nanoparticle albuminbound paclitaxel demonstrated favorable results in elderly groups. Phase III trials are now underway to confirm their therapeutic benefit in nonsquamous cell carcinoma $[19,20]$ (Table 1).

The application of combined therapy with a platinum agent is controversial. A meta-analysis and subgroup analysis of the Cochrane Database of Systematic Review on 51 randomized controlled trials showed that chemotherapeutics combined with a platinum agent extended OS compared to that of a nonplatinum agent; however, the toxicity also tended to worsen [42]. The results of the IFCT-501 phase III trial, in which combination therapy of carboplatin (CBDCA) plus PTX was compared with VNR or GEM monotherapy, showed that CBDCA plus PTX was superior in terms of OS and PFS, but the rate of treatment-related death also tended to be high (4.4\%) [15]. Therefore, the recommended therapeutic regimen for elderly population varies per country: monotherapy is recommended in Japan, while combined therapy with CBDCA is recommended in Western countries for patients who are in good general condition. Meanwhile, pooled analysis of two phase III trials, namely, MILES-3 and MILES-4, showed that combination treatment with cisplatin (CDDP) resulted in a more favorable response rate compared to monotherapy using a third-generation anticancer drug in patients with advanced lung cancer with a performance status (PS) of 0-1 and aged 70 years or older. Such results have been presented in the 2017 American Society of Clinical Oncology convention. PFS was extended in the group treated with combination CDDP, but no significant difference was observed in OS. In addition, the rates of toxicity, febrile neutropenia, and body malaise were also higher in the group treated with combination CDDP therapy [16]. The pharmacokinetics of CDDP is similar between the elderly and young patients [43]. Although evidence for the active recommendation of CDDP administration in elderly patients is lacking, CDDP should still be considered for such patients given that the drug has been shown to yield beneficial results. 
TABLE 1: Clinical trials of cytocidal anticancer drugs for elderly patients with NSCLC.

\begin{tabular}{|c|c|c|c|c|c|c|}
\hline Study, author & $n$. & Treatment & RR (\%) & OS (month) & PFS (month) & $\begin{array}{c}\text { Neutropenia } \\
\text { G3-4 (\%) }\end{array}$ \\
\hline \multicolumn{7}{|l|}{ Phase III trials } \\
\hline \multirow{2}{*}{ ELVIS [12] } & 78 & VNR & 19.7 & 6.5 & NR & NR \\
\hline & 96 & BSC & - & 4.9 & - & - \\
\hline \multirow{3}{*}{ MILES [13] } & 232 & $\mathrm{VNR}+\mathrm{GEM}$ & 21 & 7.6 & 4.8 & 18 \\
\hline & 233 & VNR & 18 & 8.8 & 4.5 & 25 \\
\hline & 233 & GEM & 16 & 6.6 & 4.3 & 8 \\
\hline \multirow{2}{*}{ WJTOG9904 [14] } & 88 & DTX & 22.7 & 14.3 & 5.5 & 82.9 \\
\hline & 91 & VNR & 9.9 & 9.9 & 3.1 & 69.3 \\
\hline \multirow{2}{*}{ IFCT-0501 [15] } & 225 & CBDCA + PTX & 27.1 & 10.3 & 6 & 48.4 \\
\hline & 226 & VNR or GEM & 10.2 & 6.2 & 2.8 & 12.4 \\
\hline \multirow{2}{*}{ MILES-3/MILES-4 [16] } & 263 & $\mathrm{CDDP}+\mathrm{GEM}$ or PEM & 15.5 & 9.6 & 4.6 & Significantly higher \\
\hline & 268 & GEM or PEM & 8.5 & 7.5 & 3 & $\begin{array}{l}\text { and more sever in } \\
\text { CDDP group. }\end{array}$ \\
\hline \multirow{2}{*}{ JCOG0803 [17] } & 139 & CDDP + DTX & 34.4 & 14.8 & 4.7 & 10.1 \\
\hline & 137 & DTX & 24.6 & 13.3 & 4.4 & 88.8 \\
\hline \multirow{2}{*}{ JCOG0207 [18] } & 63 & CDDP + DTX & 55 & 17 & 6.2 & 14.3 \\
\hline & 63 & DTX & 26.2 & 10.7 & 3.7 & 4.8 \\
\hline \multicolumn{7}{|c|}{ Subset analysis of elderly group } \\
\hline \multirow{2}{*}{ Socinski et al. [19] } & 73 & nab-PTX + CBDCA & 34 & 8 & 19.9 & 55 \\
\hline & 81 & PTX + CBDCA & 24 & 6.8 & 10.4 & 73 \\
\hline \multirow{2}{*}{ PARAMOUNT [20] } & 52 & PEM & 42 & 13.7 & 6.4 & 17 \\
\hline & 40 & Placebo & 43 & 12.1 & 3 & - \\
\hline
\end{tabular}

$\mathrm{RR}=$ response rate, $\mathrm{OS}=$ overall survival, $\mathrm{PFS}=$ progression free survival, $\mathrm{NR}=$ not reported, $\mathrm{VNR}=$ vinorelbine, $\mathrm{BSC}=$ best supportive care, $\mathrm{GEM}=$ gemcitabine, DTX = docetaxel, PTX = paclitaxel, CDDP = cisplatin, CBDCA = carboplatine, nab-PTX = nanoparticle albumin-bound paclitaxel, and PEM = pemetrexed.

4.2. EGFR Tyrosine Kinase Inhibitors. Active mutation in the epidermal growth factor receptor (EGFR) gene is highly correlated with the efficacy of EGFR tyrosine kinase inhibitors (EGFR-TKI). EGFR is among the indispensable biomarkers in selecting the therapeutic modality for NSCLC patients. Several phase III comparative studies, including NEJ002, have been conducted and have shown that EGFR-TKI is more effective than conventional cytotoxic agents as firstline therapy for EGFR mutation-positive lung cancer [44]. Although the median age of the subjects in these trials was approximately 60 years, several elderly patients were included in these studies, and subset analysis of PFS showed a trend in favor of EGFR-TKI in the elderly group [21-23].

By contrast, although only few comparative studies of EGFR-TKI targeting only elderly patients with EGFR mutation-positive lung cancer have been conducted, the results of the NEJ001 trial, which included patients with a PS of 3 or 4 and aged younger than 74 years, those with a PS of 2-4 and aged between 75 and 79 years, and those with a PS of 1-4 and aged older than 80 years, showed favorable outcome improvement in PS in most patients in the group given gefitinib (PFS: 6.5 months; OS: 17.8 months) [45]. In addition, the results of the NEJ003 trial that targeted patients with a PS of $0-2$ and older than 75 years showed a response rate of $74 \%$, a median PFS of 12.3 months, a one-year survival rate of $83.9 \%$, and a two-year survival rate of $58.1 \%$ in the group administered with gefitinib [46]. The results of a pooled analysis combining the NEJ001, NEJ002, and NEJ003 trials that limited the subjects to elderly patients older than 70 years showed that PFS was longer in the gefitinib administration group than in the chemotherapy group (CBDCA plus PTX), but no significant difference was observed for OS [47]. These results are similar to those of subgroup analyses of elderly patients in phase III comparative studies and indicate the high efficacy of EGFR-TKI as treatment for EGFR mutationpositive lung cancer, even in elderly patients with poor PS. Therefore, EGFR-TKI has been highly recommended and widely used in elderly patients both in Japan and in Western countries.

However, most trials that included only elderly patients used gefitinib, and studies using erlotinib or second- or thirdgeneration EGFR-TKI are limited. A comparative analysis between patients aged older and younger than 75 years showed that the efficacy of erlotinib is similar in the elderly group and in the younger group, and the adverse effects were manageable [48]. Meanwhile, although no comparative studies regarding the second-generation EGFR-TKI afatinib and dacomitinib and the third-generation EGFR-TKI osimertinib have been performed specifically for elderly patients, the results of subset analyses have suggested the efficacy of such 
TABLE 2: Subset analysis of elderly population in clinical trials of EGFR-TKIs or ALK inhibitors for NSCLC.

\begin{tabular}{|c|c|c|c|c|c|}
\hline Study & Treatment & Age & $n$. & HR for OS $(95 \% \mathrm{CI})$ & HR for PFS $(95 \% \mathrm{CI})$ \\
\hline \multicolumn{6}{|l|}{ (A) EGFR-TKIs } \\
\hline \multirow{2}{*}{ IPASS [21] } & \multirow{2}{*}{ Gefitinib } & $\geq 65$ & NR & NR & $0.58(0.45-0.76)$ \\
\hline & & $<65$ & NR & NR & $0.81(0.70-0.95)$ \\
\hline \multirow{2}{*}{ OPTIMAL [22] } & \multirow{2}{*}{ Erlotinib } & $\geq 65$ & 38 & NR & $0.17(0.07-0.43)$ \\
\hline & & $<65$ & 116 & NR & $0.19(0.11-0.31)$ \\
\hline \multirow{2}{*}{ EUROTAC [23] } & \multirow{2}{*}{ Erlotinib } & $\geq 65$ & 88 & NR & $0.28(0.16-0.51)$ \\
\hline & & $<65$ & 85 & NR & $0.44(0.25-0.75)$ \\
\hline \multirow{2}{*}{ LUX-Lung $3[24,25]$} & \multirow{2}{*}{ Afatinib } & $\geq 65$ & 134 & $0.73(0.43-1.21)$ & $0.64(0.39-1.03)$ \\
\hline & & $<65$ & 211 & $0.82(0.57-1.19)$ & $0.53(0.36-0.76)$ \\
\hline \multirow{2}{*}{ LUX-Lung $6[25,26]$} & \multirow{2}{*}{ Afatinib } & $\geq 65$ & 86 & $0.60(0.33-1.10)$ & $0.16(0.07-0.40)$ \\
\hline & & $<65$ & 278 & $0.87(0.64-1.20)$ & $0.30(0.21-0.43)$ \\
\hline \multirow{2}{*}{ ARCHER1050 [27] } & \multirow{2}{*}{ Dacomitinib } & $\geq 65$ & 94 & NR & $0.69(0.48-0.99)$ \\
\hline & & $<65$ & 133 & NR & $0.51(0.39-0.69)$ \\
\hline \multirow{2}{*}{ AURA3 [28] } & \multirow{2}{*}{ Osimertinib } & $\geq 65$ & 177 & NR & $0.34(0.23-0.50)$ \\
\hline & & $<65$ & 242 & NR & $0.38(0.28-0.54)$ \\
\hline \multicolumn{6}{|l|}{ (B) ALK inhibitors } \\
\hline \multirow{2}{*}{ PROFILE1014 [29] } & \multirow{2}{*}{ Crizotinib } & $\geq 65$ & 55 & NR & $0.90(0.43-1.87)$ \\
\hline & & $<65$ & 288 & NR & $0.45(0.29-0.70)$ \\
\hline \multirow{2}{*}{ ALEX [30] } & \multirow{2}{*}{ Alectinib } & $\geq 65$ & 70 & NR & $0.45(0.24-0.87)$ \\
\hline & & $<65$ & 233 & NR & $0.48(0.34-0.70)$ \\
\hline \multirow{2}{*}{ J-ALEX [31] } & \multirow{2}{*}{ Alectinib } & $\geq 75$ & 22 & NR & $0.28(0.06-1.19)$ \\
\hline & & $<75$ & 285 & NR & $0.34(0.21-0.56)$ \\
\hline
\end{tabular}

$\mathrm{HR}=$ hazard ratio, $\mathrm{OS}=$ overall survival, $\mathrm{PFS}=$ progression free survival, and NR = not reported.

drugs in elderly patients [24-28]. In particular, osimertinib, which is effective against the EGFR-T790M mutation that accounts for nearly $50 \%$ of susceptibility to EGFR-TKI, is known for its efficacy as well as high tolerability, and studies on its applicability in elderly patients are expected (Table 2).

4.3. ALK Inhibitors. Anaplastic lymphoma kinase (ALK) inhibitors, such as crizotinib, alectinib, and ceritinib, are currently developed and are among the treatment options for $A L K$ rearrangement-positive lung cancer, which accounts for $3 \%-5 \%$ of all cases of NSCLC. However, at present, no comparative study for such drug that targets only elderly patients has been performed. In a subset analysis of the PROFILE 1014 trial, 55 of the 343 patients were elderly (older than 65 years); the results of this analysis showed the efficacy of crizotinib and a prolonged PFS in the elderly group [29]. In addition, the results of a subgroup analysis of the ALEX study, which compared the efficacy between crizotinib and alectinib as first-line therapy, showed that the PFS was extended in both patients aged younger than 75 years and older than 75 years, and the extension was higher in the alectinib group compared to those in the crizotinib group. An analysis of the J-ALEX study that targeted the Japanese population also showed similar results $[30,31]$.

4.4. Angiogenesis Inhibitors. Combining angiogenesis inhibitors with cytotoxic drugs or molecular targeted drugs yields additive effects, which tends to be different in elderly patients from young patients. A subset analysis of the AVAiL phase
III trial of bevacizumab showed the efficacy of combination of cytotoxic agents with bevacizumab in patients aged older than 65 years [32]. Meanwhile, a subset analysis of the ECOG4599 trial and pooled analysis of the PointBreak trial showed no additive effect from the combination of cytotoxic agents with bevacizumab in elderly patients aged older than 70 years; however, the toxicity tended to increase [33]. In the SAiL trial, which is an observational study in Europe, the efficacy and safety of combining angiogenesis inhibitors with cytotoxic drugs or molecular targeted drugs in patients aged 70 years or older were similar to those in young patients [34]. The results of the ARIES trial, which is an observational study in the US, showed that neither the incidence of adverse events nor PFS in patients older than 75 years was different from those of young patients; however, OS was shorter in the elderly patients [35].

Concerning ramucirumab (RAM), which is an antivascular endothelial growth factor receptor- 2 antibody, the results of the REVEL phase III trial showed that OS, PFS, and response rate in the DTX plus RAM group were superior to the DTX alone group. In a subgroup analysis of the REVEL trial, the additive effect of RAM to DTX was not observed in elderly patients, and the incidence of adverse events higher than grade 3 tended to be higher in the DTX plus RAM group $[36,37]$ (Table 3 ).

Evidence for recommending anti-VEGF therapy for elderly patients with NSCLC is limited. Although anti-VEGF antibodies yield significant additive effects in conditions where VEGF is a key factor, such as metastasis to the central 
TABLE 3: Subset analysis of elderly population in clinical trials of angiogenesis inhibitors for NSCLC.

\begin{tabular}{lccccccc}
\hline Study & Age & $n$. & Treatment & OS (month) & HR for OS (95\% CI) & PFS (month) & HR for PFS (95\% CI) \\
\hline \multirow{2}{*}{ AVAiL [32] } & $\geq 65$ & 103 & CG + BEV (15 mg/kg) & NR & 0.88 & NR & 0.84 \\
& $<65$ & 248 & CG + BEV (15 mg/kg) & NR & 1.09 & NR & 0.85 \\
ECOG4599, PointBreak [33] & $\geq 75$ & 114 & BEV + CP & 9.6 & $1.10(0.74-1.60)$ & 5.6 & $0.95(0.62-1.44)$ \\
& $<75$ & 787 & BEV + CP & 13.4 & $0.76(0.66-0.87)$ & 6.1 & $0.69(0.60-79)$ \\
SAiL [34] & $>65$ & 623 & Chemotherapy + BEV & 14.6 & NR & 8.2 & NR \\
& $\leq 65$ & 1589 & Chemotherapy + BEV & 14.6 & NR & 7.6 & NR \\
ARIES [35] & $\geq 65$ & 1013 & Chemotherapy + BEV & 12.1 & $1.17(1.06-1.28)$ & 6.8 & $1.01(0.92-1.10)$ \\
& $<65$ & 954 & Chemotherapy + BEV & 14.2 & 1 & 6.4 & 1 \\
REVEL [36, 37] & $\geq 70$ & 127 & RAM + DTX & NR & $1.07(0.80-1.43)$ & NR & $0.94(0.73-1.22)$ \\
& $<70$ & 591 & RAM + DTX & NR & $0.81(0.70-0.94)$ & NR & $0.73(0.64-0.83)$ \\
\hline
\end{tabular}

$\mathrm{RR}=$ response rate, $\mathrm{OS}=$ overall survival, $\mathrm{PFS}=$ progression free survival, $\mathrm{HR}=$ hazard ratio, $\mathrm{NR}=$ not reported, $\mathrm{CG}=$ carboplatin/gemcitabine, $\mathrm{BEV}=$ bevacizumab, $\mathrm{CP}=$ carboplatin/paclitaxel, $\mathrm{DTX}=$ docetaxel, and $\mathrm{RAM}=$ ramucirumab.

TABLE 4: Subset analysis of elderly population in clinical trials of immune checkpoint inhibitors for NSCLC.

\begin{tabular}{|c|c|c|c|c|}
\hline Study & Age & $n$ & HR for OS $(95 \% \mathrm{CI})$ & HR for PFS $(95 \% \mathrm{CI})$ \\
\hline \multicolumn{5}{|l|}{ Nivolumab } \\
\hline \multirow{3}{*}{ CheckMate017 [38] } & $\geq 75$ & 29 & $1.85(0.76-4.51)$ & $1.76(0.77-4.05)$ \\
\hline & $65-74$ & 91 & $0.56(0.34-0.91)$ & $0.51(0.32-0.82)$ \\
\hline & $<65$ & 152 & $0.52(0.35-0.75)$ & $0.62(0.44-0.89)$ \\
\hline \multirow{3}{*}{ CheckMate057 [39] } & $\geq 75$ & 43 & $0.97(0.49-1.95)$ & $0.90(0.43-1.87)$ \\
\hline & $65-74$ & 200 & $0.94(0.69-1.27)$ & $0.63(0.45-0.89)$ \\
\hline & $<65$ & 339 & $0.89(0.70-1.13)$ & $0.81(0.62-1.04)$ \\
\hline \multicolumn{5}{|l|}{ Pembrolizumab } \\
\hline \multirow{2}{*}{ KEYNOTE024 [40] } & $\geq 65$ & 164 & NR & $0.90(0.43-1.87)$ \\
\hline & $<65$ & 141 & NR & $0.45(0.29-0.70)$ \\
\hline \multirow{2}{*}{ KEYNOTE010 [41] } & $\geq 65$ & 429 & $0.76(0.57-1.02)$ & NR \\
\hline & $<65$ & 604 & $0.63(0.50-0.79)$ & NR \\
\hline
\end{tabular}

$\mathrm{RR}=$ response rate, $\mathrm{OS}=$ overall survival, $\mathrm{PFS}=$ progression free survival, and $\mathrm{NR}=$ not reported.

nervous system $[49,50]$, malignant pleural effusion [51], and pericardial effusion, its use still requires caution.

4.5. Immune Checkpoint Inhibitors. Immune checkpoint inhibitors, including nivolumab, which was approved in 2015, show favorable outcomes in advanced lung cancer. Although the incidence of immune-related adverse events is higher in immune checkpoint inhibitors than conventional cytotoxic agents, the incidence of adverse events that lead to deterioration in general condition, such as anorexia, malaise, and myelosuppression, is low. Therefore, immune checkpoint inhibitors are considered safe for elderly patients. However, no clinical trial regarding the use of immune checkpoint inhibitors exclusive to elderly patients has been performed. The results of a subset analysis of the CheckMate 017 study showed that, in the group of patients aged 65-74 years, improvement in the survival rate was almost similar to that in patients younger than 65 years. Meanwhile, no efficacy was observed in patients 75 years or older. However, given the inclusion of a small number of patients in the elderly group, which affected the results, the efficacy of the treatment cannot be concluded to be inferior based on the analysis results
$[38,39]$. In addition, pembrolizumab, an anti-programmed cell death-1 (PD-1) antibody, was shown to be superior to combined therapy with a platinum agent as first-line treatment for diseases with more than 50\% programmed cell death ligand 1 (PD-L1) expression in a tumor surface. A subgroup analysis regarding PFS showed favorable results from immune checkpoint inhibitors independent of age in patients older than 65 years and those who were younger [40]. Meanwhile, the results of a subgroup analysis in the KEYNOTE-010 study, which is a comparative trial between pembrolizumab and DTX as second-line therapy, did not show significant improvement in the OS of patients aged 65 years or older (Table 4) [41].

Studies have shown that, compared to young people, alterations in the number of T cells in the elderly are minimal in immunosenescence due to aging but acquired antigenspecific immune function declines. In particular, studies have reported hastened immune aging, particularly in patients with cancer [52-54]. However, some elderly patients respond favorably to immune checkpoint inhibitors despite their age. Therefore, the mechanism by which the immune response to tumors, which is activated by immune checkpoint inhibitors, 
is affected by aging remains unclear, and further studies are necessary.

\section{Conclusion}

With an increase in the number of elderly patients with lung cancer, comprehensive assessments of problems specific to elderly patients and provision of adequate and appropriate therapy are rising concerns. A number of clinical trials targeting elderly patients are currently underway, and further evidence is anticipated.

\section{Data Availability}

All date related to this paper are available in PubMed.

\section{Conflicts of Interest}

The authors declare that there are no conflicts of interest regarding the publication of this article.

\section{Authors' Contributions}

Nakano Takayuki and Tanimura Keiko contributed equally to this work.

\section{Acknowledgments}

Nakano Takayuki and Tanimura Keiko would like to thank all the co-authors involved in this paper.

\section{References}

[1] "The top 10 causes of death - World Health Organization," http://www.who.int/mediacentre/factsheets/fs310/en/.

[2] Cancer Statistics Facts: Lung and Bronchus Cancer, Surveillance, Epidemiology, and End Results (SEER) Program of National Cancer Institute, https://seer.cancer.go.

[3] Cancer Registry and Statistics, Cancer Information Service, National Cancer Center, Japan.

[4] A. G. Sacher, L. W. Le, N. B. Leighl, and L. E. Coate, "Elderly patients with advanced NSCLC in phase III clinical trials: Are the elderly excluded from practice-changing trials in advanced NSCLC?" Journal of Thoracic Oncology, vol. 8, no. 3, pp. 366368, 2013.

[5] A. Daste, C. Chakiba, C. Domblides et al., "Targeted therapy and elderly people: A review," European Journal of Cancer, vol. 69, pp. 199-215, 2016.

[6] Centers for Disease Control and Prevention, "Chronic obstructive pulmonary disease among adults - United States, 2011," Morbidity and Mortality Weekly Report, vol. 61, no. 46, pp. 938943, 2012.

[7] K. M. M. Islam, X. Jiang, T. Anggondowati, G. Lin, and A. K. Ganti, "Comorbidity and survival in lung cancer patients," Cancer Epidemiology, Biomarkers \& Prevention, vol. 24, no. 7, pp. 1079-1085, 2015.

[8] L. Repetto, L. Fratino, R. A. Audisio et al., "Comprehensive geriatric assessment adds information to Eastern Cooperative Oncology Group performance status in elderly cancer patients: an Italian Group for Geriatric Oncology Study," Journal of Clinical Oncology, vol. 20, no. 2, pp. 494-502, 2002.

[9] E. D. Poggio, A. D. Rule, R. Tanchanco et al., "Demographic and clinical characteristics associated with glomerular filtration rates in living kidney donors," Kidney International, vol. 75, no. 10, pp. 1079-1087, 2009.

[10] E. A. Sotaniemi, A. J. Arranto, O. Pelkonen, and M. Pasanen, "Age and cytochrome P450-linked drug metabolism in humans: An analysis of 226 subjects with equal histopathologic conditions," Clinical Pharmacology \& Therapeutics, vol. 61, no. 3, pp. 331-339, 1997.

[11] T. D. Nolin, J. Naud, F. A. Leblond, and V. Pichette, "Emerging evidence of the impact of kidney disease on drug metabolism and transport," Clinical Pharmacology \& Therapeutics, vol. 83, no. 6, pp. 898-903, 2008.

[12] The Elderly Lung Cancer Vinorelbine Italian Study Group, "Effects of vinorelbine on quality of life and survival of elderly patients with advanced non-small-cell lung cancer," Journal of the National Cancer Institute, vol. 91, no. 1, pp. 66-72, 1999.

[13] C. Gridelli, F. Perrone, C. Gallo et al., "Chemotherapy for elderly patients with advanced non small-cell lung cancer: the Multicenter Italian Lung Cancer in the Elderly Study (MILES) phase III randomized trial," Journal of the National Cancer Institute, vol. 95, no. 5, pp. 362-372, 2003.

[14] S. Kudoh, K. Takeda, K. Nakagawa et al., "Phase III study of docetaxel compared with vinorelbine in elderly patients with advanced non-small-cell lung cancer: results of the West Japan Thoracic Oncology Group trial (WJTOG 9904)," Journal of Clinical Oncology, vol. 24, no. 22, pp. 3657-3663, 2006.

[15] E. Quoix, G. Zalcman, J.-P. Oster et al., "Carboplatin and weekly paclitaxel doublet chemotherapy compared with monotherapy in elderly patients with advanced non-small-cell lung cancer: IFCT-0501 randomised, phase 3 trial," The Lancet, vol. 378, no. 9796, pp. 1079-1088, 2011.

[16] A. Morabito, L. Cavanna, A. Luciani et al., " $4^{\star}$ Cisplatin in addition to single-agent first-line chemotherapy in elderly patients with advanced non-small-cell lung cancer (NSCLC): efficacy results of a joint analysis of the multicentre, randomized phase 3 MILES-3 and MILES-4 studies," Annals of Oncology, vol. 28, no. suppl_6, 2017.

[17] T. Abe, K. Takeda, Y. Ohe et al., "Randomized phase III trial comparing weekly docetaxel plus cisplatin versus docetaxel monotherapy every 3 weeks in elderly patients with advanced non-small-cell lung cancer: The intergroup trial JCOG0803/WJOG4307L," Journal of Clinical Oncology, vol. 33, no. 6, pp. 575-581, 2015.

[18] H. Tsukada, A. Yokoyama, K. Goto et al., "Randomized controlled trial comparing docetaxel-cisplatin combination with weekly docetaxel alone in elderly patients with advanced nonsmall-cell lung cancer: Japan Clinical Oncology Group (JCOG) 0207," Japanese Journal of Clinical Oncology, vol. 45, no. 1, Article ID hyul76, pp. 88-95, 2015.

[19] M. A. Socinski, C. J. Langer, I. Okamoto et al., "Safety and efficacy of weekly nab ${ }^{\circledR}$-paclitaxel in combination with carboplatin as first-line therapy in elderly patients with advanced non-small-cell lung cancer," Annals of Oncology, vol. 24, no. 2, Article ID mds461, pp. 314-321, 2013.

[20] C. Gridelli, F. De Marinis, M. Thomas et al., "Final efficacy and safety Results of pemetrexed continuation maintenance therapy in the elderly from the PARAMOUNT phase III study," Journal of Thoracic Oncology, vol. 9, no. 7, pp. 991-997, 2014. 
[21] T. S. Mok, Y.-L. Wu, S. Thongprasert et al., "Gefitinib or carboplatin-paclitaxel in pulmonary adenocarcinoma," The New England Journal of Medicine, vol. 361, no. 10, pp. 947-957, 2009.

[22] C. Zhou, Y.-L. Wu, G. Chen et al., "Erlotinib versus chemotherapy as first-line treatment for patients with advanced EGFR mutation-positive non-small-cell lung cancer (OPTIMAL, CTONG-0802): a multicentre, open-label, randomised, phase 3 study," The Lancet Oncology, vol. 12, no. 8, pp. 735-742, 2011.

[23] R. Rosell, E. Carcereny, R. Gervais et al., "Erlotinib versus standard chemotherapy as first-line treatment for European patients with advanced EGFR mutation-positive non-small-cell lung cancer (EURTAC): a multicentre, open-label, randomised phase 3 trial," The Lancet Oncology, vol. 13, no. 3, pp. 239-246, 2012.

[24] L. V. Sequist, J. C.-H. Yang, N. Yamamoto et al., "Phase III study of afatinib or cisplatin plus pemetrexed in patients with metastatic lung adenocarcinoma with EGFR mutations," Journal of Clinical Oncology, vol. 31, no. 27, pp. 3327-3334, 2013.

[25] J. C.-H. Yang, Y.-L. Wu, M. Schuler et al., "Afatinib versus cisplatin-based Chemotherapy for EGFR mutation-positive lung adenocarcinoma (LUX-Lung 3 and LUX-Lung 6): analysis of overall survival data from two randomised, phase 3 trials," The Lancet Oncology, vol. 16, no. 2, pp. 141-151, 2015.

[26] Y.-L. Wu, C. Zhou, C.-P. Hu et al., "Afatinib versus cisplatin plus gemcitabine for first-line treatment of Asian patients with advanced non-small-cell lung cancer harbouring EGFR mutations (LUX-Lung 6): an open-label, randomised phase 3 trial," The Lancet Oncology, vol. 15, no. 2, pp. 213-222, 2014.

[27] Y. L. Wu, Y. Cheng, X. Zhou et al., "Dacomitinib versus gefitinib for the first-line treatment of advanced EGFR mutation positive non-small cell lung cancer (ARCHER 1050): A randomized, open-label phase III trial," Journal of Clinical Oncology, vol. 35, no. 18_suppl, pp. LBA9007-LBA9007, 2017.

[28] T. S. Mok, W. u. Y-L, M.-J. Ahn et al., "Osimertinib or Platinum-Pemetrexed in EGFR T790M-Positive Lung Cancer," New England Journal of Medicine, vol. 376, no. 7, pp. 69-640, 2017.

[29] B. J. Solomon, T. Mok, and D.-W. Kim, "First-line crizotinib versus chemotherapy in ALK-positive lung cancer," The New England Journal of Medicine, vol. 371, no. 23, pp. 2167-2177, 2014.

[30] S. Peters, D. R. Camidge, A. T. Shaw et al., "Alectinib versus crizotinib in untreated ALK-positive non-small-cell lung cancer," The New England Journal of Medicine, vol. 377, no. 9, pp. 829-838, 2017.

[31] T. Hida, H. Nokihara, M. Kondo et al., "Alectinib versus crizotinib in patients with ALK-positive non-small-cell lung cancer (J-ALEX): an open-label, randomised phase 3 trial," The Lancet, vol. 390, no. 10089, pp. 29-39, 2017.

[32] N. B. Leighl, P. Zatloukal, J. Mezger et al., "Efficacy and safety of bevacizumab-based therapy in elderly patients with advanced or recurrent nonsquamous non-small cell lung cancer in the phase III BO17704 study (AVAiL)," Journal of Thoracic Oncology, vol. 5, no. 12, pp. 1970-1976, 2010.

[33] C. J. Langer, M. A. Socinski, J. D. Patel et al., "Isolating the Role of Bevacizumab in Elderly Patients with Previously Untreated Nonsquamous Non-Small Cell Lung Cancer," American Journal of Clinical Oncology, vol. 39, no. 5, pp. 441-447, 2016.

[34] J. Laskin, L. Crinò, E. Felip et al., "Safety and efficacy of first-line bevacizumab plus chemotherapy in elderly patients with advanced or recurrent nonsquamous non-small cell lung cancer: Safety of avastin in lung trial (MO19390)," Journal of Thoracic Oncology, vol. 7, no. 1, pp. 203-211, 2012.

[35] A. J. Wozniak, M. P. Kosty, M. Jahanzeb et al., "Clinical outcomes in elderly patients with advanced non-small cell lung cancer: Results from ARIES, a bevacizumab observational cohortstudy," Clinical Oncology, vol. 27, no. 4, pp. 187-196, 2015.

[36] E. B. Garon, T. E. Ciuleanu, O. Arrieta et al., Ramucirumab plus docetaxel versus placebo plus docetaxel for second-line treatment of stage IV non-small-cell lung cancer after disease progression on platinum-based therapy (REVEL): a multicenter, double-blind, randomized phase 3 trial, vol. 384, Lancet, London, UK, 9944 edition, 2014.

[37] E. Larkins, B. Scepura, G. M. Blumenthal et al., "U.S. food and drug administration approval summary: Ramucirumab for the treatment of metastatic non-small cell lung cancer following disease progression on or after platinum-based chemotherapy," The Oncologist, vol. 20, no. 11, pp. 1320-1325, 2015.

[38] J. Brahmer, K. L. Reckamp, P. Baas et al., "Nivolumab versus docetaxel in advanced squamous-cell non-small-cell lung cancer," The New England Journal of Medicine, vol. 373, no. 2, pp. 123-135, 2015.

[39] H. Borghaei, L. Paz-Ares, L. Horn et al., "Nivolumab versus docetaxel in advanced nonsquamous non-small-cell lung cancer," The New England Journal of Medicine, vol. 373, no. 17, pp. 16271639, 2015.

[40] M. Reck, D. Rodríguez-Abreu, A. G. Robinson et al., "Pembrolizumab versus chemotherapy for PD-L1-positive non-small-cell lung cancer," The New England Journal of Medicine, vol. 375, no. 19, pp. 1823-1833, 2016.

[41] R. S. Herbst, P. Baas, D. W. Kim et al., "Pembrolizumab versus docetaxel for previously treated, PD-L1-positive, advanced nonsmall-cell lung cancer (KEYNOTE-010): A randomized controlled trial," The Lancet, vol. 387, no. 10027, pp. 1540-1550, 2016.

[42] F. N. Santos, T. B. de Castria, M. R. S. Cruz, and R. Riera, "Chemotherapy for advanced non-small cell lung cancer in the elderly population," Cochrane Database of Systematic Reviews, vol. 10, p. CD010463, 2015.

[43] H. Minami, Y. Ohe, S. Niho et al., "Comparison of pharmacokinetics and pharmacodynamics of docetaxel and cisplatin in elderly and non-elderly patients: Why is toxicity increased in elderly patients?" Journal of Clinical Oncology, vol. 22, no. 14, pp. 2901-2908, 2004.

[44] M. Maemondo, A. Inoue, K. Kobayashi et al., "Gefitinib or chemotherapy for non-small-cell lung cancer with mutated EGFR," The New England Journal of Medicine, vol. 362, no. 25, pp. 2380-2388, 2010.

[45] A. Inoue, K. Kobayashi, K. Usui et al., "First-line gefitinib for patients with advanced non-small-cell lung cancer harboring epidermal growth factor receptor mutations without indication for chemotherapy," Journal of Clinical Oncology, vol. 27, no. 18, p. 3071, 2009.

[46] M. Maemondo, Y. Minegishi, A. Inoue et al., "First-line gefitinib in patients aged 75 or older with advanced non-small cell lung cancer harboring epidermal growth factor receptor mutations. NEJ 003 study," Journal of Thoracic Oncology, vol. 7, no. 9, pp. 1417-1422, 2012.

[47] N. Morikawa, Y. Minegishi, A. Inoue et al., "First-line gefitinib for elderly patients with advanced NSCLC harboring EGFR mutations. A combined analysis of North-East Japan Study Group studies," Expert Opinion on Pharmacotherapy, vol. 16, no. 4, pp. 465-472, 2015. 
[48] K. Kurishima, H. Satoh, T. Kaburagi et al., "Erlotinib for elderly patients with non-small cell lung cancer: Subset analysis from a population based observational study by the Ibaraki Thoracic Integrative (POSITIVE) Research Group," Molecular and Clinical Oncology, vol. 1, no. 5, pp. 828-832, 2013.

[49] A. Ilhan-Mutlu, M. Osswald, Y. Liao et al., "Bevacizumab prevents brain metastases formation in lung adenocarcinoma," Molecular Cancer Therapeutics, vol. 15, no. 4, pp. 702-710, 2016.

[50] M. A. Socinski, C. J. Langer, J. E. Huang et al., "Safety of bevacizumab in patients with non-small-cell lung cancer and brain metastases," Journal of Clinical Oncology, vol. 27, no. 31, pp. 5255-5261, 2009.

[51] K. Kitamura, K. Kubota, M. Ando et al., "Bevacizumab plus chemotherapy for advanced non-squamous non-small-cell lung cancer with malignant pleural effusion," Cancer Chemotherapy and Pharmacology, vol. 71, no. 2, pp. 457-461, 2013.

[52] P. J. Linton and K. Dorshkind, "Age-related changes in lymphocyte development and function," Nature Immunology, vol. 5, no. 2, pp. 133-139, 2004.

[53] G. Pawelec, "Immunosenescence and cancer," Biogerontology, vol. 75, no. 2, pp. 1-5, 2017.

[54] L. Tsaknaridis, L. Spencer, N. Culbertson et al., "Functional assay for human $\mathrm{CD} 44^{+} \mathrm{CD} 25^{+}$Treg cells reveals an agedependent loss of suppressive activity," Journal of Neuroscience Research, vol. 74, no. 2, pp. 296-308, 2003. 


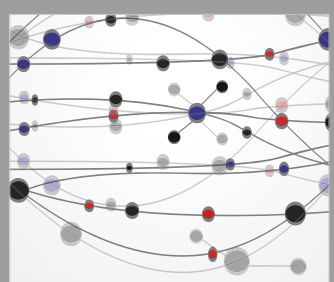

The Scientific World Journal
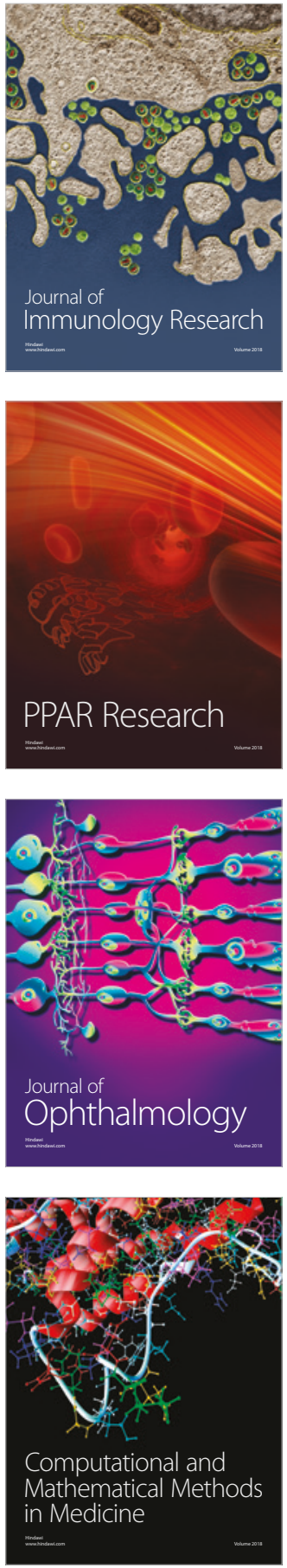

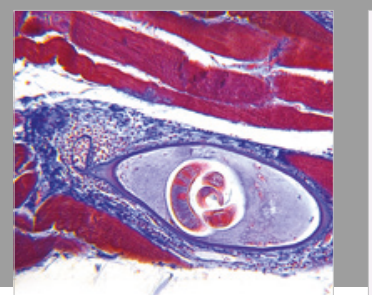

Gastroenterology Research and Practice

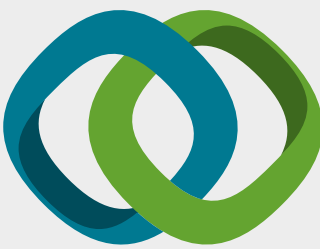

\section{Hindawi}

Submit your manuscripts at

www.hindawi.com
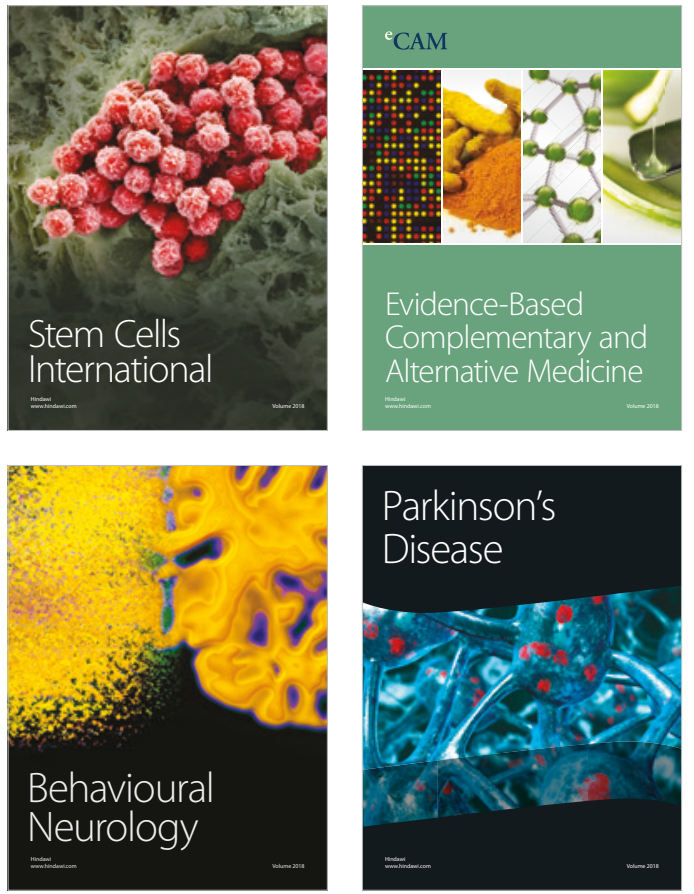

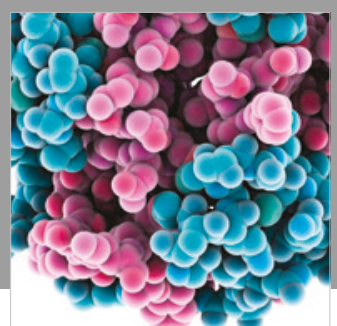

ournal of

Diabetes Research

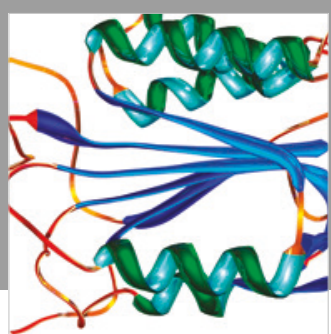

Disease Markers
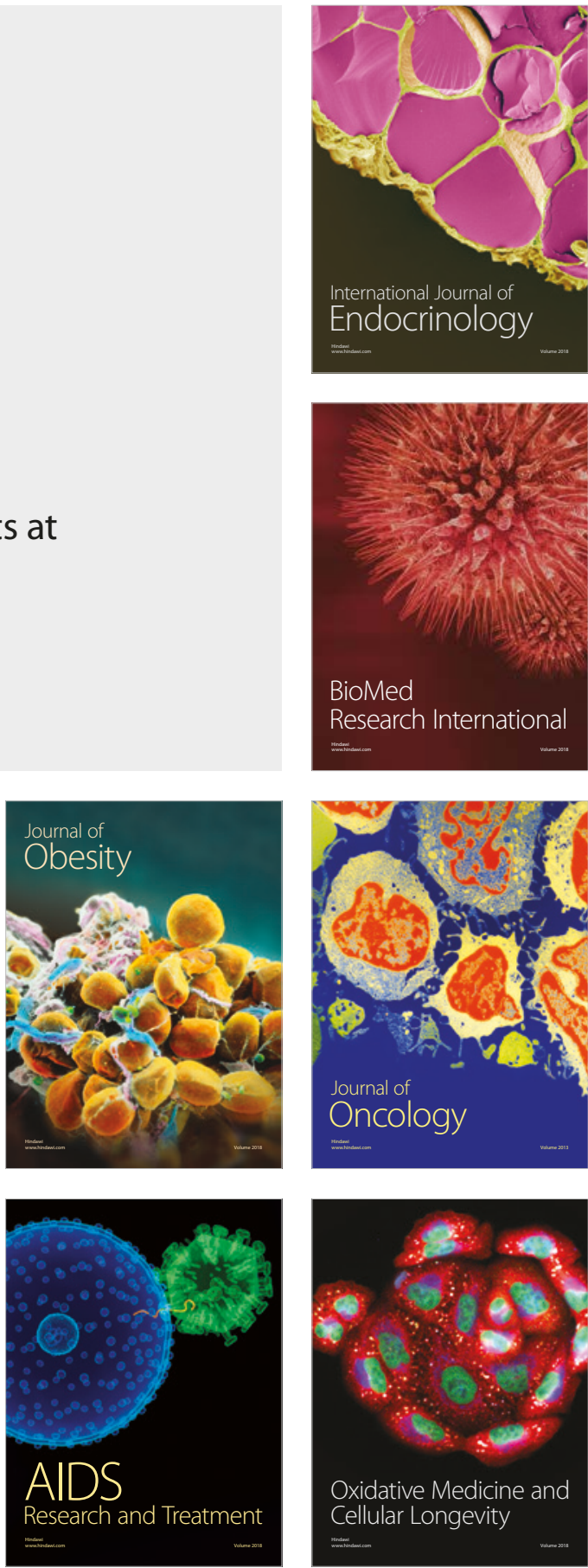